\title{
Characteristics and outcomes of patients with advanced non-small-cell lung cancer who declined to participate in randomised clinical chemotherapy trials
}

\author{
C Tanai,', H Nokihara', S Yamamoto', H Kunitoh', N Yamamoto', I Sekine', Y Ohe' and T Tamura' \\ 'Department of Medical Oncology, National Cancer Center Hospital, Tokyo, Japan; ${ }^{2}$ Cancer Information Services and Surveillance Division, Center for \\ Cancer Control and Information Services, National Cancer Center, Tokyo, Japan
}

\begin{abstract}
There are inadequate data on the outcomes of patients who declined to participate in randomised clinical trials as compared with those of participants. We retrospectively reviewed the patient characteristics and treatment outcomes of both participants and non-participants in the two randomised trials for chemotherapy-naive advanced non-small-cell lung cancer. Trial | compared four platinum-based combination regimens. Trial 2 compared two sequences of carboplatin plus paclitaxel and gefitinib therapies. Nineteen of I 19 (16\%) and 153 (37\%) patients declined to participate in Trials I and 2, respectively. Among the background patient characteristics, the only variable associated with trial participation or declining was the patients' attending physicians $(P<0.00 \mathrm{I})$. Important differences were not observed in the clinical outcomes between participants and non-participants, for whom the response rates were 30.6 vs $34.2 \%$ and the median survival times were 489 vs 461 days, respectively. The hazard ratio for overall survival, adjusted for other confounding variables, was 0.965 (95\% confidence interval: $0.73-1.28$ ). In conclusion, there was no evidence to suggest any difference in the characteristics and clinical outcomes between participants and non-participants. Trial designs and the doctor-patient relationship may have an impact on the patient accrual to randomised trials.

British Journal of Cancer (2009) I 00, 1037-1042. doi: I0.1038/sj.bjc.6604982 www.bjcancer.com
\end{abstract}

Published online 17 March 2009

(C) 2009 Cancer Research UK

Keywords: randomised clinical trial; trial participation; trial effect; lung cancer

Randomised clinical trials (RCTs) are the definitive method for comparing the efficacy of treatments and a crucial step in the development of new cancer treatments. There has always been a big problem that their low accrual rates limit their progress (Lara et al, 2001; Corrie et al, 2003; Go et al, 2006).

A number of studies have examined the motivations of patients for accepting or declining entry to RCTs (Jenkins and Fallowfield, 2000; Madsen et al, 2000, 2002; Ellis et al, 2001; Wright et al, 2004; Ho et al, 2006; Albrecht et al, 2008). The results of questionnaire surveys administered to patients regarding clinical trials revealed that two of the most common reasons for entering the trial were the hope for personal benefit and the opportunity to contribute to the research knowledge thereby benefiting others in the future (Jenkins and Fallowfield, 2000; Madsen et al, 2000, 2002; Ellis et al, 2001; Wright et al, 2004; Albrecht et al, 2008). On the other hand, the common reasons for declining participation were worries about the process of randomisation, overestimation of the benefits of standard therapy and fear of the trial's experimental nature (Jenkins and Fallowfield, 2000; Ellis et al, 2001; Ho et al, 2006).

However, inadequate data are available on the actual outcomes of non-participants compared with those participating in RCTs

* Correspondence: Dr C Tanai, Department of Internal Medicine, National Cancer Center Hospital, 5-I-I Tsukiji, Chuo-ku, Tokyo 104-0045, Japan; E-mail: ctanai@ncc.go.jp

Revised I 3 February 2009; accepted 17 February 2009; published online 17 March 2009
(Schmoor et al, 1996; Braunholtz et al, 2001; Burgers et al, 2002; Peppercorn et al, 2004; West et al, 2005). Although several reports and their review (Braunholtz et al, 2001) have suggested the existence of a 'trial effect', in which participants enjoy favourable outcomes, others, especially those which attempted to exclude the confounding factors, have refuted this finding (Schmoor et al, 1996; Burgers et al, 2002; Peppercorn et al, 2004; West et al, 2005).

On the other hand, if participation in prospective trials is associated with certain clinical characteristics of the patients, generalisability of the conclusion from the data to the clinical practise, even in patients who meet the restrictive eligibility criteria, should be in question.

The purpose of this study was to analyse the characteristics and outcomes of the patients who met the eligibility criteria but declined to participate in RCTs, as compared with those who did participate, and to search for clues to improve patient accrual to clinical trials.

\section{MATERIALS AND METHODS}

Between October 2000 and October 2005, each of the 272 patients, who fulfilled the entry criteria of our top priority studies during the period, was informed of all aspects of RCTs on non-small-cell lung cancer (NSCLC) and was invited to participate in one of the two trials to be conducted at the National Cancer Center Hospital, Tokyo, Japan. We make it a rule for each patient with advanced 
lung cancer to be hospitalised for the first-line chemotherapy. All patients are then checked for the eligibility criteria of clinical trials available at the time and recorded in our database, whether or not they are treated on trials.

Signed informed consent was obtained from the patients for future statistical analysis of their clinical courses and outcomes, even when they were treated outside clinical trials.

Trial 1 was conducted to compare the four platinum-based combination regimens (cisplatin-irinotecan, carboplatin-paclitaxel, cisplatin-gemcitabine and cisplatin-vinorelbine) in patients with untreated advanced NSCLC between October 2000 and June 2002 (Ohe et al, 2007). When patients declined to participate, cisplatin-based combination regimens, such as cisplatin-irinotecan, the reference arm of the trial, were recommended. The patients ultimately selected the treatment following discussions with their families and the physicians.

Trial 2 was conducted between June 2003 and October 2005 to compare the following two treatment arms; (A) four courses of carboplatin and paclitaxel (CP) followed by gefitinib, and (B) gefitinib until disease progression followed by $\mathrm{CP}$, in patients with advanced NSCLC (Nokihara et al, 2008). When patients declined to participate, platinum-based combination regimens, such as $\mathrm{CP}$, were recommended. The patients ultimately selected the treatment following discussions with their families and the physicians; treatment options included gefitinib as first-line chemotherapy, when the patients and their families wished to start with it.

Patients in each trial had to meet the following criteria: histologically and/or cytologically documented NSCLC; clinical stage IV or IIIB (including only patients with no indications for curative radiotherapy); no earlier systematic chemotherapy; at least one measurable lesion; age 20-74 years old; Eastern Cooperative Oncology Group Performance Status (PS) of 0 or 1; adequate haematological, hepatic and renal functions; and partial pressure of arterial oxygen of 60 torr or more. Each patient was required to submit a written informed consent before entry.

Four physicians (A, B, C and D) participated in Trial 1 and five physicians (A, B, C, D and E) in Trial 2. All were male. Physicians $A, B, C$ and $D$ had $16,14,11$ and 9 years of experience, respectively, at the time of activation of Trial 1 (October 2000), and Physician E had 9 years of experience at the start of Trial 2 (June 2003). One of the five attending staff physicians and one to two residents or trainees attended each consultation. Which doctor actually offered the RCTs depended on each case and was not recorded, but the attending staff physician finally confirmed the decision by the patient.

Paper and/or electronic medical records from the initial visit to our centre to the end of the follow-up were retrospectively reviewed. Demographic data (age, gender, smoking history), medical information (tumour histology, clinical stage, performance status, therapy characteristics), and clinical outcomes (response rate, follow-up time, overall survival time, 1- and 2 -year survival rates) were abstracted and analysed. The response was evaluated according to the Response Evaluation Criteria in Solid Tumours (RECIST) (Therasse et al, 2000) by the attending physicians. It is our policy to assess clinical responses with RECIST, even in routine practise. Follow-up time at our institution was defined as the period from the initiation of the first day of the initial therapy or decision of no therapy, to the last day at our institution (including death during follow-up). Survival data of the patients who left our institution could be collected by enquiry into official agency for family registry in Japan.

$\chi^{2}$-tests and logistic regression analysis was used to assess associations between patient characteristics and the rate of declining to participate. Overall survival (OS) curves were produced using the Kaplan-Meier method and compared with the log rank test. All participants (those who agreed to be enroled into the RCT) and non-participants (those who declined to participate in the RCT) were included in the OS analysis. A Cox proportional hazards model was used to adjust for other potential confounding factors (age, gender, smoking history, clinical stage and PS) in comparing the OS of participants and non-participants. $P$-values $<0.05$ were considered statistically significant. The data collected were analysed using an SPSS II statistical package.

Japanese ethics guidelines for clinical and epidemiological studies, which took effect in August 2007, do not mandate institutional review board (IRB) approval for a single-institutional, retrospective data analysis from the medical charts, when the predesignated person of the institution so judges. This study was thus exempted from ethical review of IRB in due process, on the judgment of the responsible official, deputy director of National Cancer Center Hospital.

\section{RESULTS}

There were no significant differences in the outcomes between the arms of each trial. In Trial 1, no statistically significant differences in the response rate, progression-free survival and OS were observed between the four regimens. In Trial 2, there were no statistically significant differences in the median survival time (MST) (18.8 and 17.2 months) and the survival rate at 1 year between the two arms. Seventy-five patients declined to participate in those trials, and 1 of the 197 who initially accepted entry withdrew consent, refusing to continue the trial immediately after randomisation.

Table 1 shows the patient characteristics and rate of declining. 100 patients accepted and 19 patients (16\%) declined entry to Trial 1, and 96 patients accepted and 57 patients (37\%) declined entry to clinical Trial 2 (including the one patient already mentioned who withdrew consent after randomisation) $(P<0.001)$. No significant influence on the rate of declining of patient gender, age,

Table I Patient characteristics and rate of declining

\begin{tabular}{|c|c|c|c|c|c|c|c|c|c|}
\hline & \multicolumn{3}{|c|}{ Clinical trial I } & \multicolumn{3}{|c|}{ Clinical trial 2} & \multicolumn{3}{|c|}{ Total } \\
\hline & $\mathbf{P}$ & NP & ROD (\%) & $\mathbf{P}$ & $\mathbf{N P}$ & ROD (\%) & $\mathbf{P}$ & $\mathbf{N P}$ & ROD (\%) \\
\hline No. & 100 & 19 & 16 & 96 & 57 & 37 & 196 & 76 & 28 \\
\hline \multicolumn{10}{|l|}{ Gender } \\
\hline Male & 64 & 12 & 16 & 55 & 34 & 38 & 119 & 46 & 28 \\
\hline Female & 36 & 7 & 16 & 41 & 23 & 36 & 77 & 30 & 28 \\
\hline \multicolumn{10}{|l|}{ Age } \\
\hline$<60$ & 46 & 9 & 16 & 37 & 29 & 44 & 83 & 38 & 31 \\
\hline$\geqslant 60$ & 54 & 10 & 16 & 59 & 28 & 32 & 113 & 38 & 25 \\
\hline \multicolumn{10}{|c|}{ Smoking history } \\
\hline+ & 69 & 9 & 12 & 55 & 33 & 38 & 124 & 43 & 26 \\
\hline- & 31 & 10 & 24 & 41 & 24 & 37 & 72 & 33 & 31 \\
\hline \multicolumn{10}{|c|}{ Clinical stage } \\
\hline III & 24 & 6 & 20 & 21 & 19 & 48 & 45 & 25 & 36 \\
\hline IV & 76 & 13 & 15 & 75 & 38 & 34 & $|5|$ & 51 & 25 \\
\hline \multicolumn{10}{|l|}{ PS } \\
\hline 0 & 27 & 4 & 13 & 47 & 19 & 29 & 74 & 23 & 24 \\
\hline I & 73 & 15 & 17 & 49 & 38 & 44 & 122 & 53 & 30 \\
\hline \multicolumn{10}{|l|}{ Physicians } \\
\hline A & 32 & 5 & 14 & 23 & 25 & 52 & 55 & 30 & 35 \\
\hline B & 28 & 0 & 0 & 25 & I & 4 & 53 & 1 & 2 \\
\hline C & 18 & 2 & 10 & 34 & 4 & 11 & 52 & 6 & 10 \\
\hline$D$ & 22 & 12 & 35 & 7 & 18 & 72 & 29 & 30 & 51 \\
\hline$E$ & - & - & - & 7 & 9 & 56 & 7 & 9 & 56 \\
\hline
\end{tabular}

Abbreviations: $N P=$ non-participants, $P=$ participants; $P S=$ performance status; $\mathrm{ROD}=$ rate of declining. 
Table 2 Prediction of participation or declining to trials

\begin{tabular}{|c|c|c|c|c|}
\hline & \multicolumn{2}{|c|}{ Univariate analysis $^{\mathbf{a}}$} & \multicolumn{2}{|c|}{ Multivariate analysis ${ }^{\mathbf{b}}$} \\
\hline & Odds ratio $(95 \% \mathrm{Cl})$ & $P$-value & Odds ratio $(95 \% \mathrm{Cl})$ & $P$-value \\
\hline Age $(<60$ vs $\geqslant 60)$ & $0.735(0.432-1.250)$ & 0.254 & $0.701(0.376-1.310)$ & 0.266 \\
\hline Smoking history (+vs -) & $1.394(0.815-2.386)$ & 0.225 & $2.538(1.162-5.541)$ & 0.019 \\
\hline Clinical stage (III vs IV) & $0.608(0.339-1.089)$ & 0.093 & $0.681(0.346-1.340)$ & 0.266 \\
\hline PS $(0$ vs I) & $1.398(0.792-2.467)$ & 0.247 & $0.785(0.396-1.554)$ & 0.487 \\
\hline
\end{tabular}

Abbreviations: $\mathrm{NP}=$ non-participant; $\mathrm{P}=$ participant; $\mathrm{PS}=$ performance status; $\mathrm{ROD}=$ rate of declining. ${ }^{\mathrm{a} B y}$ Pearson's $\chi^{2}$-test. ${ }^{\mathrm{b}}$ By logistic regression analysis.

Table 3 Number of courses of the first-line chemotherapy

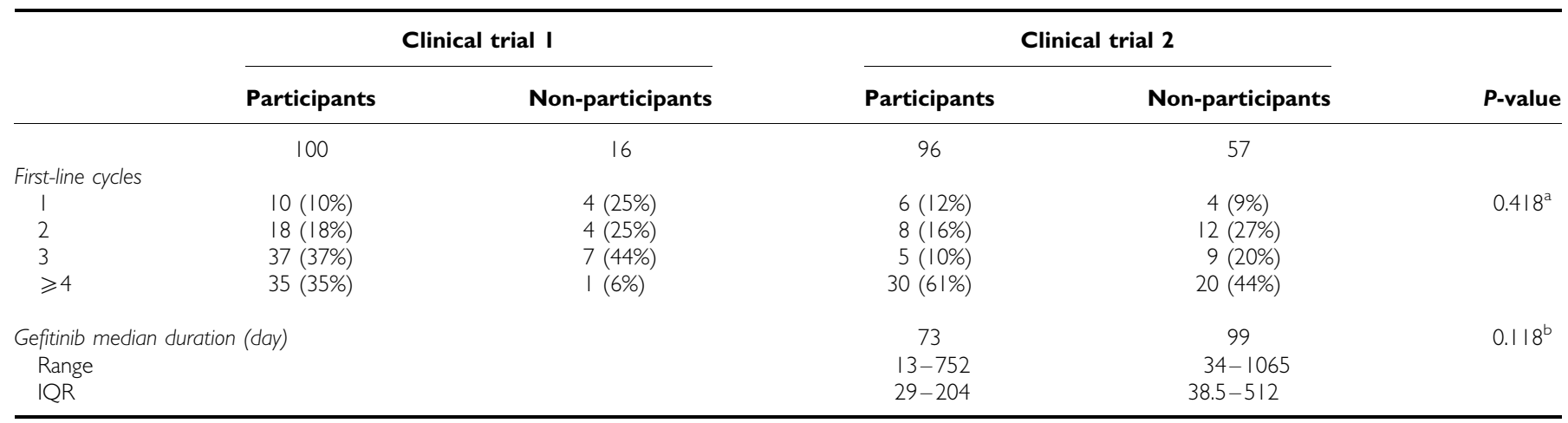

Abbreviation: $\mathrm{IQR}=$ interquartile range. ${ }^{\text {aB }}$ P Pearson's $\chi^{2}$-test. ${ }^{\mathrm{b}}$ By log rank test.

smoking history, tumour histology, clinical stage or PS was observed (Table 2). There were, however, large differences in the rates of decline among the attending physicians who informed the patients about the trials and asked them to participate $(P<0.001)$.

The treatment regimens for those who declined participation in the clinical trials were as follows. The majority of those who declined participation in Trial 1 selected one of the four platinumbased combination regimens presented in the trial: cisplatinirinotecan 4, cisplatin-vinorelbine 3, cisplatin-gemcitabine 1, carboplatin-paclitaxel 4 . Three patients in Trial 1 desired to have no more active treatments and opted for supportive care only, but later received active treatment at their referred hospitals. The detail of their therapy is unknown.

The majority of those who declined participation in Trial 2 selected carboplatin-based combination chemotherapy: carboplatin-paclitaxel 34 and carboplatin-gemcitabine 11, there by reflecting the shift to carboplatin for advanced NSCLC in Japan at the time of Trial 2, on the basis of the reports on the activity of the carboplatin-based regimens (Kelly et al, 2001; Schiller et al, 2002; Ohe et al, 2007). Twelve patients $(21 \%)$ selected gefitinib as first-line chemotherapy.

Survival was analysed for all of the 196 participants and 76 of the non-participants. Post-therapy was analysed for all of the 196 participants and 73 of the non-participants, who were treated at our centre. There was one possible treatment-related death due to perforation of the colon during gefitinib treatment in Trial 2. No other toxic deaths were observed among either participants or non-participants. More participants of both the clinical trials were given four cycles or more of the firstline chemotherapy, probably reflecting protocol regulations (Table 3).

Table 4 summarises the treatment after the initial therapy. There were no significant differences between participants and nonparticipants in the number of chemotherapy regimens. Six (8\%) of
Table 4 Treatment after the first-line chemotherapy

\begin{tabular}{lccc}
\hline & $\begin{array}{c}\text { Participants } \\
\text { 196 (\%) }\end{array}$ & $\begin{array}{c}\text { Non-participants } \\
\text { 73 (\%) }\end{array}$ & $\boldsymbol{P}$-value $^{\mathbf{a}}$ \\
\hline Chemotherapy regimen & & & \\
$0^{\mathrm{b}}$ & 26 & 40 & 0.108 \\
$1^{2}$ & 38 & 26 & \\
3 & 22 & 25 & \\
$>4$ & 9 & 8 & \\
Radiotherapy & 5 & 1 & \\
Pleural or pericardial drainage & 19 & 34 & 0.031 \\
Operation on metastatic brain & 1 & 5 & 0.227 \\
tumors & & 3 & 0.122 \\
Early-phase trials & 13 & 8 & 0.300 \\
\hline
\end{tabular}

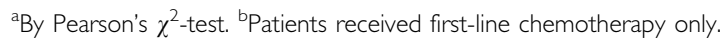

those who declined participation in the trial later participated in early-phase clinical trials of experimental therapies.

We have observed no clinically relevant differences in the clinical outcomes between participants and non-participants (Table 5). Clinical response to the initial therapy was analysed for all of the 196 participants and 73 of the non-participants, excluding three patients who were not treated at our institute. The response rate was $30.6 \%$ in participants and $34.2 \%$ in non-participants $(P=0.325)$. The median follow-up time at our centre was 388 days for participants and 406 days for non-participants, which was not statistically different.

The OS was not different between participants and nonparticipants (Table 5 and Figure 1), with a hazard ratio of participants $v s$ non-participants of 0.998 ( $95 \%$ confidence interval: $0.76-1.32)$. No significant difference in OS was observed either in Trial 1 (Figure 2) or in Trial 2 (Figure 3). 
Table 5 Clinical outcomes

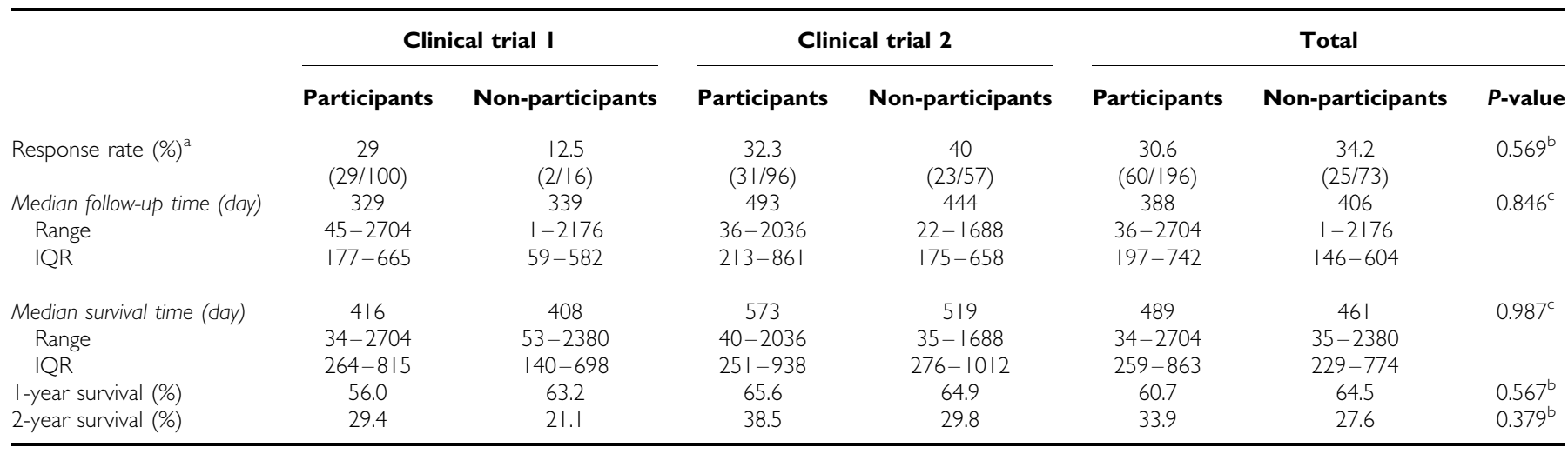

Abbreviation: $\mathrm{IQR}=$ interquartile range. ${ }^{\mathrm{a} E x c l u d i n g}$ three patients who did not receive active treatment at our center. ${ }^{\mathrm{b}}$ By Pearson's $\chi^{2}$-test. 'By log rank test.

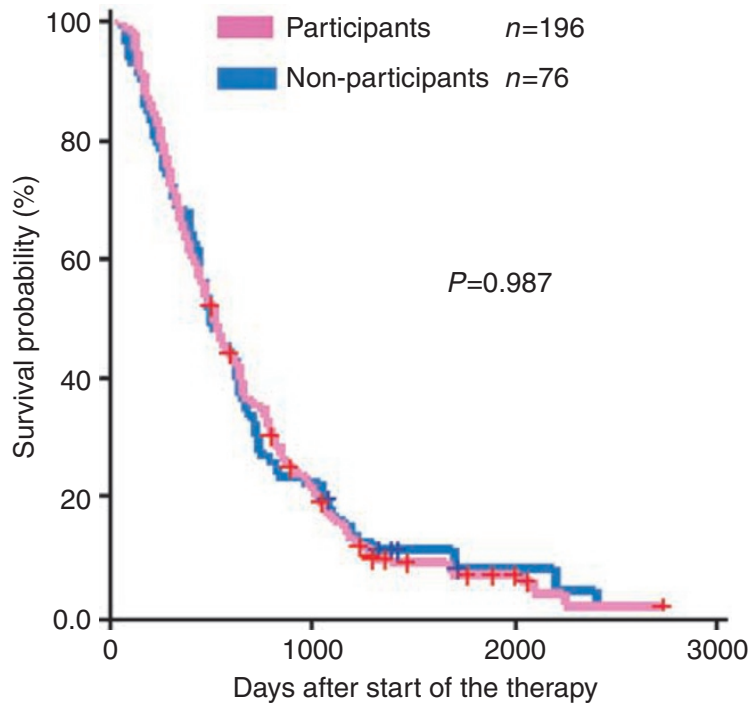

Figure I Overall survival of those who declined to participate in randomised trials (blue line, $n=76$ ) as compared with the participants (pink line, $n=196)$. No significant difference can be observed.

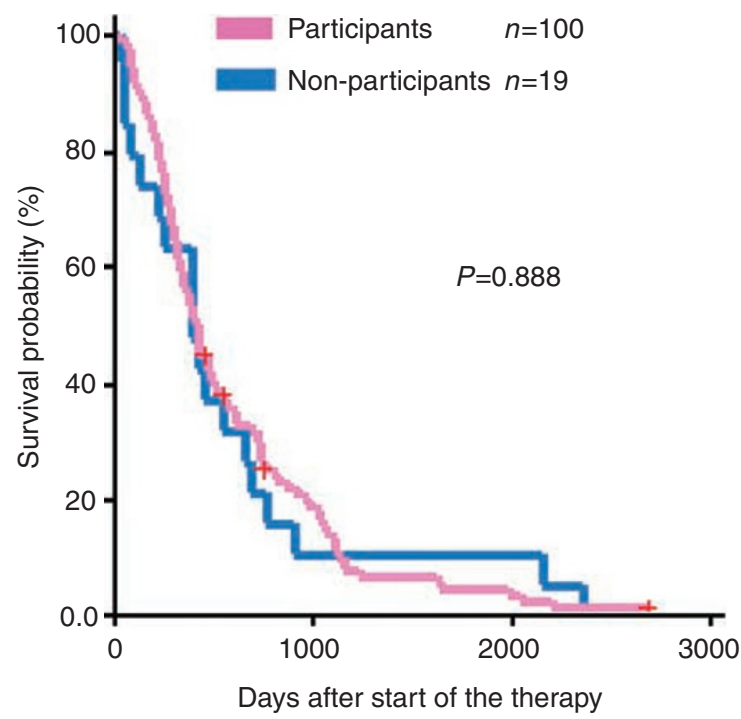

Figure 2 Overall survival of those who declined to participate in Trial I (blue line, $n=19$ ) as compared with the participants (pink line, $n=100$ ). No significant difference can be observed.

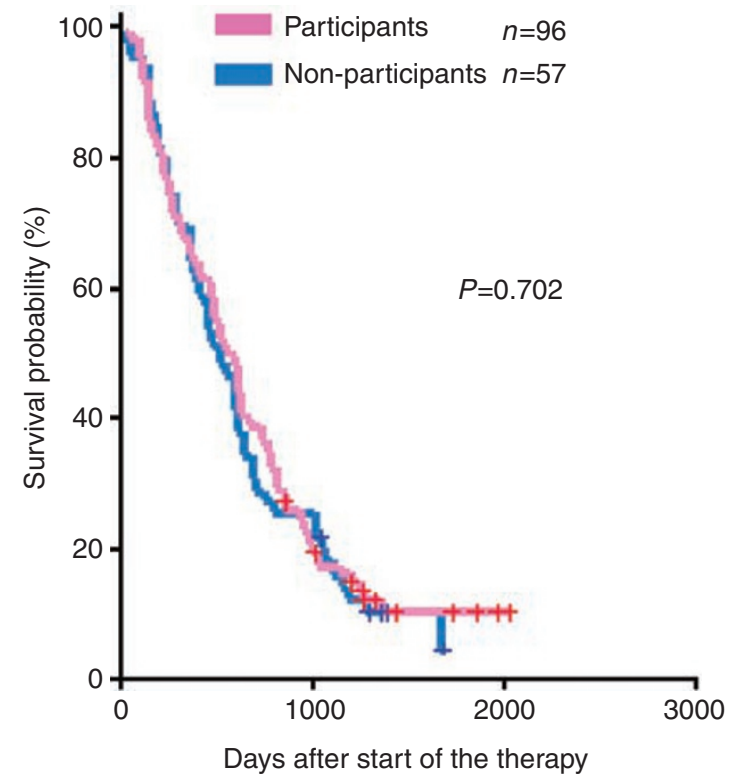

Figure 3 Overall survival of those who declined to participate in Trial 2 (blue line, $n=57$ ) as compared with the participants (pink line, $n=96$ ). No significant difference can be observed.

With the Cox proportional hazards model adjusted for gender, age, smoking history, clinical stage and PS, the hazard ratio of participants $v s$ non-participants was 0.965 (95\% confidence interval: $0.73-1.28, P=0.805)$. Among the patient characteristics, PS was the only significant factor associated with OS in multivariate analysis $(P=0.006$, by Cox proportional model).

\section{DISCUSSION}

It has been argued that trial participants have better outcomes than those who are not enroled in clinical trials. Several investigations have reported a favourable overall trend with trial entry (Braunholtz et al, 2001; Peppercorn et al, 2004; West et al, 2005). This 'trial effect' could derive from several factors, such as protocol effect (the way treatments are delivered), care effect (extra care related to data gathering), Hawthorne effect (changes in doctor or patient behaviour on the basis of the knowledge that they are under observation) or placebo effect (psychologically mediated benefits) (Braunholtz et al, 2001; Peppercorn et al, 2004).

In majority of the reports comparing outcomes between participants and non-participants of clinical trials, however, the 
non-participant 'controls' were chosen from differently pooled database, which could include baseline imbalances between groups and hindsight bias (Davis et al, 1985; Braunholtz et al, 2001; Peppercorn et al, 2004). In this study, we compared the characteristics and outcomes of those who met the eligibility criteria but declined to participate in randomised trials, and instead chose to receive standard therapy. We thus aimed at excluding confounding factors as much as possible.

On the other hand, physician triage is pointed out to be one of the barriers to cancer clinical trial accrual (Lara et al, 2001; Corrie et al, 2003; Go et al, 2006; Ho et al, 2006). We excluded the barrier by making it a rule to offer clinical trials to every patient with advanced NSCLC who satisfied the eligibility criteria.

The response rate, MST, 1-year and 2-year survival rates were all similar in both groups. We have to admit that response evaluation might not be as strict in off-protocol therapy. However, the hazard ratio for the OS was very close to 1 . Although the confidence interval of 0.73 to 1.28 could not rule out the existence of clinically important difference in the treatment effect, it could not by any means be taken as a clinically relevant prognostic factor. We thus believe this confidence interval of the adjusted hazard ratio, 0.73 1.28 , was narrow enough to justify the conclusion that the clinical outcomes of trial participants and non-participants were not different in our study. The differences in the number of cycles of chemotherapy given to participants and non-participants may suggest the so-called protocol effect (Braunholtz et al, 2001; Peppercorn et al, 2004), in which explicit careful description of treatment regimens could lead to improvement of outcomes. On the other hand, there clearly existed no 'care effect' representing the differences in incidental aspects of treatment or care between participants and non-participants, which the protocol may require, such as extra follow-up or extra nursing care (Braunholtz et al, 2001; Peppercorn et al, 2004). In our cases, the same treatment teams took charge of and followed both groups of patients in the same manner, and found no differences in the post-treatment characteristics or follow-up periods. Thus, our first finding was that the clinical trials themselves seemed to have no influence on the outcomes or pattern of care of the patients.

The second finding was that we could not find any demographic characteristics to influence the patients' willingness to participate in clinical trials. Taken together with the first finding, both the characteristics and outcomes of the non-participants were very similar to the participants. This would imply that the participants ably represented the whole patient population of the disease status who met the eligibility criteria, and that conclusions from the clinical trials could be generalised.

Our study, however, could only show the similarity in the prognosis of the participants and non-participants, and, unlike an earlier report (Link et al, 1986), not that of the treatment effect itself. This could not be evaluated because there were no significant differences in the clinical effect between the arms in both Trial 1 and Trial 2. If newer, much more effective experimental treatment were presented in the trials, the outcome could be better in trial participants, which was the case in the adjuvant chemotherapy trial for osteosarcoma (Link et al, 1986). In that report, eligible patients who declined randomisation, but were given adjuvant chemotherapy, also had better outcomes. Therefore, a very effective treatment could lead to a better outcome both on and off trial. Ideally, strict comparison of the effects of the study participation itself would require randomised design of the trial participation (Braunholtz et al, 2001; Peppercorn et al, 2004), which is almost impossible to conduct.

Thirdly, the declining rate seemed to be influenced by the trial design. Trial 1 was the comparison of four similar platinumdoublet regimens. On the other hand, Trial 2 was the comparison of two arms with sequentially different types of chemotherapy. In general, people might have the impression that injection therapy would be more effective, and less convenient, than oral administration. It is easy to understand that more patients felt difficulty in accepting the randomisation of different types of therapy, such as Trial 2 (Schmoor et al, 1996; Jenkins and Fallowfield, 2000).

The declining rate also seemed to be greatly affected by the attending physician. The attending physician with longer experience as a thoracic oncologist tended to have lower rate of declination. Even though we do not have records on who actually informed the participants regarding the trial, residents or trainees under Physician A seemed to have had more chance to lead the consultation, which might have affected the rate of declination. Trust in the doctor is one of the most important reasons for agreeing to enter an RCT, whereas it has also been cited as the main reason for declining to participate (Jenkins and Fallowfield, 2000; Ellis et al, 2001; Stryker et al, 2006). Patients prefer the doctor to make the treatment decisions rather than to be randomised. A recent report emphasises the influence of physicians' clinical communication on patients' decision-making on participation in clinical trials (Albrecht et al, 2008). Improving communication and more interventions by clinical research coordinators and other medical staff members in all eligible patients may improve the accrual rate (Fallowfield et al, 1998; Wright et al, 2004; Stryker et al, 2006).

Finally, it was interesting to find that $8 \%$ of those who declined the RCTs participated in early-phase trials during follow-up. It is possible that the lack of effective therapies had changed their recognition of clinical trials. However, it might support the psychological states of patients as reported in earlier studies (Jenkins and Fallowfield, 2000; Ellis et al, 2001; Wright et al, 2004); patients expect experimental therapies to give them improved effectiveness but with fear of uncertainty. They are reported to have negative opinions regarding the principle of randomisation. Better understanding of the patients' decision-making process and the factors influencing their psychological states may lead to improvement in RCT accrual.

Our study has several limitations. One is that it was conducted at a single academic institution; the situation might well have been different in others or when the research was performed on a multiinstitution basis. The second is that we analysed data from only two trials and could not definitely conclude that a trial design would affect the patient accrual. Third, we have no data on the reasons for patient participation. That information would be definitely useful for analysing factors for consent or declining to participate, and would help to improve the accrual rate. Further research is required.

In conclusion, there was no evidence of any difference in the response rates and survival times between participants and nonparticipants. The declining rate of clinical trials was influenced by the referring physicians and trial designs. Further analysis of the decision-making process of those offered trials is warranted, for it may improve patient accrual to RCTs.

\section{REFERENCES}

Albrecht TL, Eggly SS, Gleason MEJ, Harper FWK, Foster TS, Peterson AM, Orom H, Penner LA, Ruckdeschel JC (2008) Influence of clinical communication on patients' decision making on participation in clinical trials. J Clin Oncol 26: 2666-2673

Braunholtz DA, Edwards SJL, Lilford RJ (2001) Are randomized clinical trials good for us (in the short term)? Evidence for a 'trial effect'. J Clin Epidemiol 54: $217-224$
Burgers JA, Arance A, Ashcroft L, Hodgetts J, Lomax L, Thatcher N (2002) Identical chemotherapy schedules given on and off trial protocol in small cell lung cancer response and survival results. Br J Cancer 87: $562-566$

Corrie P, Shaw J, Harris R (2003) Rate limiting factors in recruitment of patients to clinical trials in cancer research: descriptive study. BMJ 327: $320-321$ 
Davis S, Wright P, Schulman SF, Hill LD, Pinkham RD, Johnson LP, Jones TW, Kellogg HB, Radke HM, Sikkema WW, Jolly PC, Hammar SP (1985) Participants in prospective, randomized clinical trials for resected nonsmall cell lung cancer have improved survival compared with nonparticipants in such trials. Cancer 56: 1710-1718

Ellis PM, Butow PN, Tattersall MHN, Dunn SM, Houssami N (2001) Randomized clinical trials in oncology: understanding and attitudes predict willingness to participate. J Clin Oncol 19: 3554-3561

Fallowfield LJ, Jenkins V, Brennan C, Sawtell M, Moynihan C, Souhami RL (1998) Attitudes of patients to randomised clinical trials of cancer therapy. Eur J Cancer 34: 1554-1559

Go RS, Frisby KA, Lee JA, Mathiason MA, Meyer CM, Ostern JL, Walther SM, Schroeder JE, Meyer LA, Umberger KE (2006) Clinical trial accrual among new cancer patients at a community-based cancer center. Cancer 106: $426-433$

Ho J, Pond GR, Newman C, Maclean M, Chen EX, Oza AM, Siu LL (2006) Barriers in phase I cancer clinical trials referrals and enrollment: fiveyear experience at the Princess Margaret Hospital. BMC Cancer 6: 263

Jenkins V, Fallowfield L (2000) Reasons for accepting or declining to participate in randomized clinical trials for cancer therapy. $\mathrm{Br} J$ Cancer 82: $1783-1788$

Kelly K, Crowley J, Bunn PA, Presant CA, Grevstad PK, Moinpour CM, Ramsey SD, Wozniak AJ, Weiss GR, Moore DF, Israel VK, Livingston RB, Gandara DR (2001) Randomized phase III trial of paclitaxel plus carboplatin versus vinorelbine plus cisplatin in the treatment of patients with advanced non-small cell lung cancer: a Southwest Oncology Group Trial. J Clin Oncol 19: 3210-3218

Lara PN, Higdon R, Lim N, Kwan K, Tanaka M, Lau DHM, Wun T, Welborn J, Meyers FJ, Christensen S, O'Donnell R, Richman C, Scudder SA, Tuscano J, Gandara DR, Lam KS (2001) Prospective evaluation of cancer clinical trial accrual patterns: identifying potential barriers to enrollment. J Clin Oncol 19: $1728-1733$

Link MP, Goorin AM, Miser AW, Green AA, Pratt CB, Belasco JB, Pritchard J, Malpas JS, Baker AR, Kirkpatrick JA, Ayala AG, Shuster JJ, Abelson HT, Simone JV, Vietti TJ (1986) The effect of adjuvant chemotherapy on relapse-free survival in patients with osteosarcoma of the extremity. N Engl J Med 314: 1600 - 1606

Madsen SM, Holm S, Davidsen B, Munkholm P, Schlichting P, Riis P (2000) Ethical aspects of clinical trials: the attitudes of participants in two non-cancer trials. J Intern Med 248: 463-474
Madsen SM, Mirza MR, Holm S, Hilsted KL, Kampmann K, Riis P (2002) Attitudes towards clinical research amongst participants and nonparticipants. J Intern Med 251: 156-168

Nokihara H, Ohe Y, Yamada K, Kawaishi M, Kato T, Yamamoto N, Sekine I, Kunitoh H, Saijo N, Tamura T (2008) Randomized phase II study of sequential carboplatin/paclitaxel (CP) and gefitinib (G) in chemotherapy-naïve patients with advanced non-small-cell lung cancer (NSCLC): final results. J Clin Oncol 26: 441s (Suppl; abstr 8069)

Ohe Y, Ohashi Y, Kubota K, Tamura T, Nakagawa K, Negoro S, Nishiwaki Y, Saijo N, Ariyoshi Y, Fukuoka M (2007) Randomized phase III study of cisplatin plus irinotecan versus carboplatin plus paclitaxel, cisplatin plus gemcitabine, and cisplatin plus vinorelbine for advanced non-smallcell lung cancer: Four-Arm Cooperative Study in Japan. Ann Oncol 18: 317-323

Peppercorn JM, Weeks JC, Cook EF, Joffe S (2004) Comparison of outcomes in cancer patients treated within and outside clinical trials: conceptual framework and structured review. Lancet 363: 263-270

Schiller JH, Harrington D, Belani CP, Langer C, Sandler A, Krook J, Zhu J, Johnson DH (2002) Comparison of four chemotherapy regimens for advanced non-small-cell lung cancer. $N$ Engl J Med 346: $92-98$

Schmoor C, Olschewski M, Schumacher M (1996) Randomized and nonrandomized patients in clinical trials: experiences with comprehensive cohort studies. Stat Med 15: 263-271

Stryker JE, Wray RJ, Emmons KM, Winer E, Demetri G (2006) Understanding the decisions of cancer clinical trial participants to enter research studies: factors associated with informed consent, patient satisfaction, and decisional regret. Patient Educ Couns 63: 104-109

Therasse P, Arbuck SG, Eisenhauer EA, Wanders J, Kaplan RS, Rubinstein L, Verweij J, Glabbeke MV, Oosterom AT, Christian MC, Gwyther SG (2000) New guidelines to evaluate the response to treatment in solid tumors. J Natl Cancer Inst 92: 205-216

West J, Wright J, Tuffnell D, Jankowicz D, West R (2005) Do clinical trials improve quality of care? A comparison of clinical processes and outcomes in patients in a clinical trial and similar patients outside a trial where both groups are managed according to a strict protocol. Qual Saf Health Care 14: 175-178

Wright JR, Whelan TJ, Schiff S, Dubois S, Crooks D, Haines PT, DeRosa D, Roberts RS, Gafni A, Pritchard K, Levine MN (2004) Why cancer patients enter randomized clinical trials: exploring the factors that influence their decision. J Clin Oncol 22: 4312-4318 